\title{
One wave or another in the pandemic: psychological well-being during the third COVID-19 wave
}

\section{BACKGROUND}

After a year from the emergence of COVID-19 in February 2020, between March and May 2021 Italy faced its third wave of infections. Previous studies have shown that in the first phases of the pandemic certain factors had a protective effect against distress. However, little is known regarding the role of possible protective variables after prolonged pandemic situations.

\section{PARTICIPANTS AND PROCEDURE}

454 Italian adults were asked questions regarding the intensity of mental states and emotions experienced, the perceived usefulness of lockdown, the feeling of living a normal life, and the coping strategies implemented to face the pandemic.

\section{RESULTS}

Our findings indicate that the presence of acceptance attitudes toward restrictive measures and the implementation of recreational activities helped participants face a prolonged pandemic with positive emotions.

\section{CONCLUSIONS}

These results extend previous findings about the impact of several behavioral variables on individuals' mental states and emotions experienced during the COVID-19 pandemic in Italy.

KEY WORDS

COVID-19; lockdown; psychological well-being; emotions

organization - 1: Department of Dynamic and Clinical Psychology, and Health Studies, La Sapienza University

of Rome, Rome, Italy · 2: Department of Brain and Behavioral Sciences, University of Pavia, Pavia, Italy

AUthors' COntributions - A: Study design - B: Data collection - C: Statistical analysis - D: Data interpretation .

E: Manuscript preparation · F: Literature search · G: Funds collection

Corresponding Author - Michela Marchetti, Department of Dynamic and Clinical Psychology, and Health Studies,

La Sapienza University of Rome, Via degli Apuli 1, 00185 Rome, Italy, e-mail: michela.marchetti@uniroma1.it

to Cite this ARTICLE - Marchetti, M., Gatti, D., Inguscio, L., \& Mazzoni, G. (2022). One wave or another in the pandemic:

psychological well-being during the third COVID-19 wave. Health Psychology Report, 10(1), 13-19. https://doi.org/

10.5114/hpr.2022.113383

RECEIVED 07.11.2021 · REVIEWED 08.12.2021 · ACCEPTED 16.01.2022 · PUBLISHED 15.02.2022 


\section{BACKGROUND}

On December $1^{\text {st }}, 2019$, the first diagnosis of COVID-19 infection in China was made (Huang et al., 2020). Within a few months, several cases emerged across the world, making the COVID-19 outbreak a pandemic disease. Since the first wave of COVID-19 infections, which affected a large number of countries approximately between March 2020 and June 2020, other COVID-19 waves have been reported. This pe-

Michela Marchetti, Daniele Gatti, Lucio Inguscio, Giuliana Mazzoni riod deeply affected people's psychological well-being, interfering with their daily functioning (Groarke et al., 2020).

To date, Italy has faced three waves of COVID-19: the first one between March and June 2020, the second one between October and December 2020 and finally the third one between March and May 2021. During the first wave, a full and nation-wide lockdown was applied from March to May 2020, then the measures were lifted around June 2020, and then, from November 2020, selected restrictions were applied, classifying the Italian regions in color zones depending on the severity of the outbreak (from white, with low rates of infection and severe cases, to red, with very high rates). No studies so far have been conducted with the aim of investigating the factors that can help maintain positive emotions during prolonged lockdown measures due to repeated waves of the pandemic.

Given the relevance of this period for the entire humanity, scientific research extensively studied the physical and psychological reactions to what people have been experiencing. Overall, one of the most studied topics in this pandemic is "fear of COVID-19", an emotion arising due to its rapid spread and high mortality rate (e.g., Dymecka et al., 2021). It was reported by Dymecka and colleagues (2021) that fear of COVID-19 and perceived stress were negatively related to life satisfaction and, in agreement with previous studies (Zhang et al., 2020), the researchers concluded that stress negatively influences people's well-being.

Another interesting finding on COVID-19 fear concerns its link with physical activity. Wilczyńska and colleagues (2021) found that people undertook physical activity to face the fear of COVID-19, to manage the stressful situation and escape the daily routine.

In addition to physical activity, general recreational activities are very important for coping with stress, providing opportunities to bring people together and implement socialization and communication. However, we need to note that also other behavioral variables, such as thoughts and feelings experienced during this period, can be relevant as protective factors.

Several studies indeed showed that the development of coping strategies and the experience of positive emotions were facilitated by several protective factors such as social support, sport, sharing thoughts and feelings about COVID-19 with others, as well as perceiving the usefulness of restrictive measures (Armour et al., 2020; Golemis et al., 2021; Marchetti et al., in press).

Previous studies indicated that the perceived usefulness of lockdown positively affected people's emotions, suggesting that better communication by the government of the experimental findings supporting the political decisions made can enhance people's well-being. Similarly, the results suggested that also the behavioral measures devised by the government and tailored to the need to increase people's commitment to the proposed rules are crucial in increasing the well-being of the population (Marchetti et al., in press). However, such results were limited to the first wave. The first wave had different characteristics compared to subsequent waves occurring over time, as it was characterized by the novelty and surprise of the experience, the hope of a relatively rapid solution, and the desire to activate coping strategies (people were singing or playing from windows and balconies, for example). Subsequent waves, occurring after months, lack these characteristics, while triggering a greater sense of hopelessness, of helplessness and the inability to see an end to the pandemic (Johansson et al., 2021). Here, we aimed to investigate the third wave of the pandemic in Italy, occurring more than one year after the first. Specifically, we were interested in examining how, during the third wave, people's emotions and mental states were affected by behavioral variables such as the perceived usefulness of lockdown, the feeling of living a normal life, and various coping strategies implemented to face the pandemic. Indeed, as the days in the pandemic situation went by, people's feelings and experiences significantly changed (Johansson et al., 2021) and it is relevant to assess how people's well-being was affected during this period.

The predictors were tailored to measure three relevant spheres of people's life. The first predictor - the perceived usefulness of lockdown - was intended to measure people's opinion on the decision taken by the government in order to counter the spread of the virus; the second predictor - the feeling of living a normal life - was intended to measure people's internal perceptions regarding the period they were living in; while the third predictor - the coping strategies implemented to face the pandemic - was intended to measure external mechanisms and everyday actions that people put in place during those days.

The main goal of the present study was thus to extend to subsequent waves of the pandemic the research on the effect of COVID-19 of elements that might protect people's psychological health (i.e., mental states and emotions experienced during this period). 
Table 1

Distribution of participants by age group and gender

\begin{tabular}{lcccccc}
\hline & \multicolumn{3}{c}{ Age } & \multirow{2}{*}{ Total } \\
\hline \multirow{2}{*}{ Gender } & $<25$ & $26-35$ & $36-50$ & $>51$ & \\
& Women & 88 & 74 & 66 & 115 & 343 \\
& Men & 27 & 18 & 18 & 48 & 111 \\
Total & 115 & 92 & 84 & 163 & 454 \\
\hline
\end{tabular}

\section{PARTICIPANTS AND PROCEDURE}

\section{PARTICIPANTS}

Four hundred and fifty-four Italian adults volunteered to participate in the experiment (343 women and 111 men; age range $=19-77$ years, $M=41.00$, $S D=16.00$, median $=43.00$, mode $=60.00)$. One hundred and sixteen were under 25 years of age, 91 were between 26 and 35 years, 84 were between 36 and 50 years, and 163 were over 51 . Table 1 shows the distribution of the individuals by gender and age groups. Twenty-nine percent of the sample were unemployed, twenty-six percent were students, thirty-five percent were full-time workers, two percent were laid off workers, sixteen percent were freelance workers, and forty-two percent were retired (Table 2).

Informed consent was obtained from all participants before the experiment. The study was approved by the Department Ethics Committee of La Sapienza University of Rome (protocol no. 0000292 del 01/04/2020 [UOR: SI000092 - Classif. VII/15]).

\section{MATERIALS AND PROCEDURE}

Participants were contacted and tested during the period of severe restrictions when many regions in Italy became "Red Zones" between March and April 2021. The survey was developed on Google Forms and the recruitment was conducted through social media. The invitation and link for the study was sent via email, WhatsApp, Facebook, and other social media, throughout the entire nation.

After providing informed consent and demographic information, participants were asked to answer several questions regarding: i) the intensity of various mental states and emotions experienced, ii) the perceived usefulness of lockdown, iii) the feeling of living a normal life, and iv) the frequency of use of coping strategies implemented to face the pandemic.

In the first part of the questionnaire administered to the participants, which is based on the Brief Mood Introspection Scale (BMIS; Mayer \& Gaschke, 2001) and the Brunel Mood Scale (BRUMS; Terry et al., 2003), the
Table 2

Distribution of participants by job

\begin{tabular}{lc}
\hline & Total $(N=454)$ \\
\hline Education, $n(\%)$ & $29(6.4)$ \\
Unemployed & $120(26.4)$ \\
Student & $159(35.0)$ \\
Full-time worker & $12(2.6)$ \\
Laid off worker & $75(16.5)$ \\
Freelancer & $59(13.0)$ \\
Retired &
\end{tabular}

intensity of the mental states and the emotions experienced was investigated by asking participants to rate the intensity of the following items: active, energetic, resilient, curious, determined, supportive, compassionate, anxious, fearful, vulnerable, oppressed, unaffectionate, unsentimental, indifferent, hostile, lonely. Each adjective represents the answer to a question such as "How do you feel now?", "How have you been feeling in the past week, including today?", or "How have you been feeling?". The participant has to select the intensity of mood (through items/adjectives) by selecting an option from a rating scale from 0 (not at all) to 4 (extremely). The first seven items were considered to have a positive emotional valence, and the others to have a negative emotional valence.

The other three parts of the questionnaire were aimed respectively at measuring people's opinion on the decision taken by the government in order to counter the spread of the virus, people's internal perceptions regarding the normality of their life, and external actions that they put in place during the third wave of COVID-19 in Italy.

The perceived usefulness of lockdown was measured by asking participants the following questions: "Is staying at home useful for you?", "Are you experiencing something new?". The answers were on a 5-level Likert scale; they ranged from 0 (not at all) to 4 (extremely). Lockdown has led many individuals
Psychological well-being during the third COVID-19 wave in Italy 
to explore new areas of interest, reassess their lives, their role in society, the importance of family members and friends. In this sense lockdown has also provided the opportunity for some novel experiences, which are investigated in the questions.

The feeling of living a normal life was investigated by asking participants the following questions: "Can you stay focused?", "Do you study/work easily?", "Is it easy to maintain old social relationships?", "Is it easy to develop new social relationships?", "Do you feel deprived of your freedom?". The answers were on a 5-level Likert scale; they ranged from 0 (not at all) to 4 (extremely).

Finally, the coping strategies implemented were investigated by asking participants to indicate which of the following common coping strategies they put into practice to face the pandemic: "talking with friends", "playing with electronic equipment", "singing/playing instruments", "doing physical activities/ sports", "reading”, "watching television/movies", "cooking/baking". The answers were on a 5-level Likert scale; they ranged from 0 (never) to 4 (always).

\section{RESULTS}

All the analyses were performed using $\mathrm{R}$ (version 3.6.3; R Core Team, 2020). Data were analyzed through a mixed-effects approach, which incorporates both fixed effects and random effects (associated with participants and task stimuli) and allows for the specification of predictors at both the participant and/or item level. Linear mixed models (LMMs) were estimated using the lme4 R package (Bates et al., 2015).

Our main dependent variable was the intensity of the mental states and the emotions experienced by the participants. The three continuous predictors in our statistical model were i) perceived usefulness of lockdown, ii) feeling of living a normal life, iii) coping strategies implemented to face the pandemic.

Firstly, we performed an exploratory factor analysis to investigate how the selected emotions aggregated. Extraction method was set as minimum residuals and rotation was set as oblimin, the number of factors was based on parallel analysis and the threshold for factor loadings was set as .40 (Stevens, 1992). Using these parameters we identified four factors, specifically:

- A negative-internal one, comprising: anxious, fearful, vulnerable, oppressed.

- A negative-external one, comprising: unaffectionate, unsentimental, indifferent, hostile.

- A positive-internal one, comprising: active, energetic, resilient, curious, determined.

- A positive-external one, comprising: supportive, compassionate.

The emotion lonely was not found to aggregate to a factor.
For each of the four factors we estimated an LMM including all possible interactions between fixed effects. Participants and type of emotion were set as random intercepts.

In particular, in the lme4 syntax (Bates et al., 2015), the model tested was:

Intensity of emotions Perceived usefulness of lockdown $\times$ Feeling of living a normal life $\times$ Coping strategies $+(1 /$ Participant $)+(1 \mid$ Emotion $)$

For each of the four models, we then performed a model selection using the MuMIn R package, with the dredge function (Barton, 2020). This procedure selects the best fitting model (i.e., the one with lowest Akaike information criterion (AIC), which returns an estimation of the quality of the model; Akaike, 1973) fitting all possible combinations of the fixed effects included.

For the negative-internal factor, the best model identified by the model selection included the feeling of living a normal life, while the other predictors were dropped. The model refitted after the model criticism procedure included 2220 observations (49 were removed), pseudo- $R^{2}$ (total) $=.55$, pseudo- $R^{2}$ (marginal) $=.16$. Specifically, the effect of the feeling of living a normal life was significant, $t(456.82)=-12.78$, $p<.001, b=-.79$, indicating that the more participants felt they were living a normal life, the lower was the intensity of the emotions in the negativeinternal factor.

For the positive-internal factor, the best model identified by the model selection included the perceived usefulness of lockdown and the feeling of living a normal life, while the other predictor was dropped. The model refitted after the model criticism procedure included 2228 observations (42 were removed), pseudo $-R^{2}($ total $)=.57$, pseudo- $R^{2}($ marginal $)=.20$. Specifically, the effects of both perceived usefulness of lockdown, $t(451.65)=4.33, p<.001, b=.23$, and feeling of living a normal life, $t(453.36)=10.43, p<.001, b=.71$, were significant, indicating that the higher their values were, the higher was the intensity of participants' emotions in the positive-internal factor.

For the negative-external factor, the best model identified by the model selection included the feeling of living a normal life, while the other predictors were dropped. The model refitted after the model criticism procedure included 1786 observations (29 were removed), pseudo- $R^{2}$ (total) $=.54$, pseudo- $R^{2}$ (marginal) $=.13$. Specifically, the effect of the feeling of living a normal life was significant, $t(451.51)=-11.21$, $p<.001, b=-.73$, indicating that the more participants felt that they were living a normal life, the lower was the intensity of the emotions in the negativeexternal factor.

For the positive-external factor, the model selection dropped all the fixed effects predictors, as the random-effect model was the one with the lowest AIC. 


\section{DISCUSSION}

In the present study we investigated in an Italian sample how several behavioral factors might affect people's psychological health. Participants were asked to answer several questions regarding the intensity of the mental states and the emotions experienced, as well as other factors, such as: i) the perceived usefulness of lockdown, ii) the feeling of living a normal life, and iii) the coping strategies implemented to face the pandemic. The results showed that the more participants felt they were living a normal life, the lower was the intensity of the emotions in the negative-internal factor (anxious, fearful, vulnerable, oppressed) and in the negative-external factor (unaffectionate, unsentimental, indifferent, hostile). Moreover, the higher the participants' perceived usefulness of lockdown was and the stronger the feeling of living a normal life, the higher was the intensity of their emotions in the positive-internal factor (active, energetic, resilient, curious, determined). No variable predicted positive emotions towards others (positive external factor, supportive, compassionate).

These findings suggest that the intensity of negative emotions, either internal or external, is lower when participants seem to be able to continue living a normal life, even when forced to stay at home, thus curtailing their social life by avoiding in-person direct contact with others. Conversely, the intensity of positive emotions increases when participants experience the usefulness of the lockdown. In this case too, however, experiencing a level of normalcy in everyday life, in spite of the lockdown and the pandemic, increases positive emotions.

Overall, feeling being able to maintain a normal pattern of everyday activities turns out to be the best predictor of low negative emotions and increased positive internal emotions. People feel less anxious and fearful, and more energetic and resilient.

It is well known that various factors can undermine well-being, including perceived risk, which is negatively correlated with well-being (Cheng et al., 2006). Pèrez-Fuentes and colleagues (2020) focused on analyzing the emotional states resulting from the perception of the risk of being infected with COVID-19. The results showed that the perception of the risk from COVID-19 was positively correlated with negative emotional states such as sadness, depression, anxiety, anger and hostility. The authors argued that a negative emotional state increased both the perception of the risk from COVID-19 and the negative mood.

Our results add to these data, showing that people can cope with the greater perception of risk and with the fear and anxiety generated by a pandemic. A greater sense of normalcy helped people to cope with the fear and anxiety triggered by the realization that the pandemic was to stay, and the virus is to stay, and could again spread easily among the population.
Linked to this, our data also show that an important role is also played by perceiving the lockdown as a useful measure, protective against contagion. Considering the lockdown as being useful predicts stronger positive feelings, and a greater ability to cope with the contingent situation (more resilient, more energetic, more active). At the time, news, social networks and government guidelines were telling citizens that the world was halting any social activity. As documented by WHO reports, the disruption of daily routines as a result of the restrictions driven by the government guidelines added stress to many people's work and family lives (e.g., school closures that force parents to attend home school while working from home themselves), increasing the rate of depression, loneliness, self-harm, suicidal behavior and increased consumption of alcohol and drugs (WHO, 2020). The results that emerge from the study presented in this paper again complement data showing the disruptive force of lockdown on psychological well-being, as they reveal how important the possibility to understand and accept the usefulness of a lockdown can be. It follows that it is crucial, not just as a measure of social policy, but also for the psychological wellbeing of the population, that governments provide a clear, correct communication of the aims of a lockdown, which conveys the need to implement policies that protect citizens, and the society as a whole, from a clear danger, even if it necessarily limits personal liberties.

The present findings also extend the results of recent studies, which focused on the adherence to quarantine restrictions and recommendations. Marchetti and colleagues (in press) also found that during the initial lockdown the perceived usefulness of lockdown measures positively affected people's emotions, suggesting that behavioral measures devised to increase people's commitment to the proposed societal rules are crucial in order to enhance people's wellbeing. While in the Marchetti and colleagues (in press) study the best model explaining the data included only perceived usefulness of lockdown measures, in the present study on the third wave of COVID-19 we included in the model all factors examined.

Critically, instead of evaluating only data relating to mental distress in the Italian population during the pandemic (e.g., Rossi et al., 2020), our findings highlight the positive effects of the ability to adapt to the new situation, and become able to move from a state of emergency to a state of new normality, experiencing the new life-style 'as usual'. In line with this, Restubog and colleagues (2020) argued that emotional regulation is the adaptive key to cope with lifestyle changes resulting from the pandemic, since it can reduce negative mental states and strengthen coping strategies for greater psychological well-being. It is important to note, in this respect, how the role of coping strategies and 'life as usual' changes between
Psychological well-being during the third COVID-19 wave in Italy 
the first (no role) and the third phase (a crucial role), probably indicating greater psychological complexity of the moment experienced in the third phase, and probably underlining the development (or strengthening) of protective behaviors by the population in the period between the two phases.

It remains to be seen whether active coping strategies helped during the third wave of the pandemic. Previous studies (Stieg, 2020) showed that recreational activities used as coping strategies play a fundamental role to face the pandemic. However, singing, watching movies, talking with friends, and similar activities that were examined in our study, were not found to be significant in our analyses. Nonetheless, we do not think their role should be completely dismissed. In a previous study, also on the Italian population, it was found that implementing positive activities helped reduce negative emotions, in particular internal emotions, and reduce fear and anxiety, while at the same time increasing positive emotions, such as resilience, as well as the level of energy experienced (Marchetti et al., in press). While in the literature it is typically examined how positive emotions promote coping in stress situations (Moskowitz et al., 2021), in that study it was highlighted how coping helps promote positive emotions in conditions of elevated stress. That study, however, was carried out during the first wave of the pandemic in Italy, which was extremely severe. In that case people were actively coping with the emergency and the acute level of stress by organizing balcony or window meetings, collective singing, collective shows of solidarity, as well as individual activities, such as cooking, daily physical exercise, and much else. During the third wave these activities were much less frequent, and the sense of emergency had probably changed into a sense of normality of a new lifestyle. In other words, it is likely that during the third wave the sense of heroic emergency of the first wave had given way to a 'new normality', and it is such a sense of normality that was now related to a more positive view of the current situation, and to greater wellbeing. During the initial emergency, in order to reduce the intensity of negative emotions, one needed to implement coping strategies such as recreational activities to face the dangerous novelty of the pandemic, and the new experience of a curtailed lifestyle. Such need no longer existed when greater knowledge was acquired about the virus, information on potentially effective vaccines was communicated, and people realized that the virus would probably become endemic and one would need to learn to live with it.

Even if the study provides important information on how people cope with a pandemic, there are potential limitations. For example, the sample might not be homogeneous for sex and age, and this could make the study not fully generalizable. Moreover, the measurements of emotions were assessed using self-reports, which may not accurately reflect participants' emotional status. However, the topic investigated here can be very promising for future research, as it complements the vision on psychological wellbeing in a pandemic scenario, trying to present additional information on how people react, in a positive way, to a pandemic and a lockdown. It shows that important information can be acquired when the aim of research is not exclusively focused on the negative effects of a pandemic, making it possible to complete the picture of wellbeing during a prolonged pandemic event.

In conclusion, the present study shows that the presence of acceptance attitudes toward restrictive measures and the experience of regained 'normalcy' can help overcome moments of difficulty, and experience increased positive emotions and decreased negative ones. Considering previous results of the literature and our results together, we would argue that knowing the emotional and cognitive effects of the lockdown on the population would help to increase measures which facilitate the acceptance of regulations aimed at protecting the health of citizens.

\section{DATA AVAILABILITY STATEMENT}

The data analyzed in the present study are available here: https://github.com/danielegatti23/Psychological-well-being-COVID-19-third-wave-Italy-

\section{References}

Akaike, H. (1973). Information theory and an extension of the maximum likelihood principle. In B. N. Petrov \& F. Csaki (Eds.), Proceedings of the 2nd International Symposium on Information Theory (pp. 267-281). Akademiai Kiado.

Armour, C., McGlinchey, E., Butter, S., McAloneyKocaman, K., \& McPherson, K. E. (2021). The COVID-19 psychological wellbeing study: Understanding the longitudinal psychosocial impact of the COVID-19 pandemic in the UK: a methodological overview paper. Journal of Psychopathology and Behavioral Assessment, 43, 174-190. https:// doi.org/10.1007/s10862-020-09841-4

Bartoń, K. (2020). MuMIn: Multi-Model Inference. $R$ package version 1.43.17. Retrieved from https:// CRAN.R-project.org/package $=$ MuMIn

Bates, D., Maechler, M., Bolker, B., \& Walker, S. (2015). Fitting linear mixed-effects models using Ime 4. Journal of Statistical Software, 67, 1-48. https://doi. org/10.18637/jss.v067.i01

Cheng, S. K., Chong, G. H., Chang, S. S., Wong, C. W., Wong, C. S., Wong, M. T., \& Wong, K. C. (2006). Adjustment to severe acute respiratory syndrome (SARS): Roles of appraisal and post-traumatic 
growth. Psychology and Health, 21, 301-317. https://doi.org/10.1080/14768320500286450

Dymecka, J., Gerymski, R., \& Machnik-Czerwik, A. (2021). Fear of COVID-19 as a buffer in the relationship between perceived stress and life satisfaction in the Polish population at the beginning of the global pandemic. Health Psychology Report, 9, 149-159. https://doi.org/10.5114/hpr.2020.102136

Golemis, A., Voitsidis, P., Parlapani, E., Nikopoulou, V. A., Tsipropoulou, V., Karamouzi, P., Giazkoulidou, A., Dimitriadou, A., Kafetzopoulou, C., Holeva, V., \& Diakogiannis, I. (2021). Young adults' coping strategies against loneliness during the COVID-19-related quarantine in Greece. Health Promotion International, daab053. https://doi.org/ 10.1093/heapro/daab053

Groarke, J. M., Berry, E., Graham-Wisener, L., McKennaPlumley, P. E., McGlinchey, E., \& Armour, C. (2020). Loneliness in the UK during the COVID-19 pandemic: Cross-sectional results from the COVID-19 psychological well-being study. PLoS One, 15, e0239698. https://doi.org/10.1371/journal.pone.0239698

Huang, C., Wang, Y., Li, X., Ren, L., Zhao, J., Hu, Y., Zhang, L., Fan, G., Xu, J., Gu, X., Cheng, Z., Yu, T., Xia, J., Wei, Y., Wu, W., Xie, X., Yin, W., Li, H., Liu, M., ... Cao, B. (2020). Clinical features of patients infected with 2019 novel coronavirus in $\mathrm{Wu}$ han, China. The Lancet, 395, 497-506. https://doi. org/10.1016/S0140-6736(20)30183-5

Johansson, F., Côté, P., Hogg-Johnson, S., \& Skillgate, E. (2021). Depression, anxiety and stress among Swedish university students during the second and third waves of COVID-19: a cohort study. Scandinavian Journal of Public Health, 49, 750-754. https://doi.org/10.1177/14034948211031402

Marchetti, M., Gatti, D., Inguscio, L., \& Mazzoni, G. (in press). Psychological well-being and lockdown: a study on an Italian sample during the first COVID-19 wave. Psychology Hub.

Mayer, J. D., \& Gaschke, Y. N. (2001). Brief Mood Introspection Scale (BMIS). Psychology, 19, 19951995.

Moskowitz, J. T., Cheung, E. O., Freedman, M., Fernando, C., Zhang, M. W., Huffman, J. C., \& Addington, E. L. (2021). Measuring positive emotion outcomes in positive psychology interventions: a literature review. Emotion Review, 13, 60-73. https://doi.org/10.1177/1754073920950811

Pérez-Fuentes, M. D. C., Molero Jurado, M. D. M., Martos Martínez, Á., \& Gázquez Linares, J. J. (2020). Threat of COVID-19 and emotional state during quarantine: Positive and negative affect as mediators in a cross-sectional study of the Spanish population. PLoS One, 15, e0235305. https:// doi.org/10.1371/journal.pone.0235305

R Core Team (2020). R: a language and environment for statistical computing. Retrieved from https:// www.R-project.org/
Restubog, S. L. D., Ocampo, A. C. G., \& Wang, L. (2020). Taking control amidst the chaos: Emotion regulation during the COVID-19 pandemic. Journal of Vocational Behavior, 119, 103440. https://doi. org/10.1016/j.jvb.2020.103440

Rossi, R., Socci, V., Talevi, D., Mensi, S., Niolu, C., Pacitti, F., Di Marco, A., Rossi, A., Siracusano, A., \& Di Lorenzo, G. (2020). COVID-19 pandemic and lockdown measures impact on mental health among the general population in Italy. Frontiers in Psychiatry, 11, 790. https://doi.org/10.3389/fpsyt.2020.00790

Stevens, W. P. (1992). Hierarchical factor analysis and the derivation of phylogenetic skull shape characters in canids. The Paleontological Society Special Publications, 6, 281-281.

Stieg, C. (2020). When it's all too much, here's how to quell coronavirus anxiety, according to experts. Retrieved from https://www.cnbc.com/2020/03/13/ how-to-stay-calm-amid-coronavirus pandemicanxiety-relieftips.html

Terry, P. C., Lane, A. M., \& Fogarty, G. J. (2003). Construct validity of the Profile of Mood States-Adolescents for use with adults. Psychology of Sport and Exercise, 4, 125-139. https://doi.org/10.1016/ S1469-0292(01)00035-8

Wilczyńska, D., Li, J., Yang, Y., Fan, H., Liu, T., \& Lipowski, M. (2021). Fear of COVID-19 changes the motivation for physical activity participation: PolishChinese comparisons. Health Psychology Report, 9, 138-148. https://doi.org/10.5114/hpr.2021.105007

World Health Organization (2020). Emergency Committee regarding the outbreak of novel coronavirus (2019-nCoV). Coronavirus disease 2019 (COVID-19) situation reports. Retrieved from https://www.who. int/emergencies/diseases/novel-coronavirus-2019/ situation-reports

Zhang, X., Wang, Y., Lyu, H., Zhang, Y., Liu, Y., \& Luo, J. (2020). The influence of COVID-19 on well-being. PsyArXiv. https://doi.org/10.31234/osf.io/znj7h
Psychological well-being during the third COVID-19 wave in Italy 